\title{
Reaction Wheel Installation Deviation Compensation for Overactuated Spacecraft with Finite-Time Attitude Control
}

\author{
Aihua Zhang, ${ }^{1}$ Jianfei Ni, ${ }^{1}$ and Hamid Reza Karimi ${ }^{2}$ \\ ${ }^{1}$ College of Engineering, Bohai University, Jinzhou 121013, China \\ ${ }^{2}$ Department of Engineering, Faculty of Engineering and Science, The University of Agder, 4898 Grimstad, Norway \\ Correspondence should be addressed to Aihua Zhang; jsxinxi_zah@163.com
}

Received 11 October 2013; Accepted 24 October 2013

Academic Editor: Xiaojie Su

Copyright (c) 2013 Aihua Zhang et al. This is an open access article distributed under the Creative Commons Attribution License, which permits unrestricted use, distribution, and reproduction in any medium, provided the original work is properly cited.

\begin{abstract}
A novel attitude tracking control scheme is presented for overactuated spacecraft to address the attitude stabilization problem in presence of reaction wheel installation deviation, external disturbance and uncertain mass of moment inertia. An adaptive sliding mode control technique is proposed to track the uncertainty. A Lyapunov-based analysis shows that the compensation control law can guarantee that the desired attitude trajectories are followed in finite-time. The key feature of the proposed control strategy is that it globally asymptotically stabilizes the system, even in the presence of reaction wheel installation deviation, external disturbances, and uncertain mass of moment inertia. The attitude track performance using the proposed finite-time compensation control is evaluated through a numerical example.
\end{abstract}

\section{Introduction}

In present, nearly all of the highly accurate slewing maneuvers necessitate the use of nonlinear differential equations for the kinematics and dynamics during the control system design [1]. However, the attitude tracking problem is further complicated by the external disturbance and uncertain mass of moment inertia. To address these issues, there have been several important developments in the design of feedback control laws for spacecraft maneuvering. A number of control design approaches using adaptive control $[2,3]$, sliding mode control [4-7], $H_{\infty}[8,9]$, optimal control [10-13], and data driven control [14-16] have been proposed. However, few of them focus on the reaction wheel installation deviation that are of great theoretical and practical interest. In fact, the installation deviation is a widespread phenomenon, such as the actuator misalignment which is limited by the installation technique or generated by materials deforms the vehicle violent vibration during the launching process. In the area of actuator misalignment compensation, there currently exist few unified frameworks for the design of simple control structures.

Several solutions to actuator installation deviation have been presented in the literature [17-20]. In [17], the authors presented a general adaptive tracking attitude controller design framework for a spacecraft subject to the actuator installation minor angle deviation. In [18], an adaptive attitude tracking method is proposed to compensate the actuator misalignment of nearly 15 degree. And in [19], a novel algorithm is employed precisely to estimate the information, such as installation angle of wheel and CMG alignments. And then the controller design can be on for the estimation information. Moreover, another recent paper in [20] proposed an adaptive control approach for satellite formation flying, in which backstepping technique is used to synthesize a controller to handle thrust magnitude error and misalignment. However, the torque is different between thruster and reaction wheel, one is literal and the other is time-variable. That is to say that this control strategy is not suitable for reaction wheel installation deviation compensation for overactuated spacecraft attitude control.

Treating the uncertain mass of moment inertia caused by the reaction wheel misalignment is another impossibly avoided problem. In practice, in order to ensure the reliability of on-orbit spacecraft operation, especially under high altitude sever external environment, overactuatation is widely employed to guarantee the control system reliability service. And finite-time is meanwhile necessary for time 


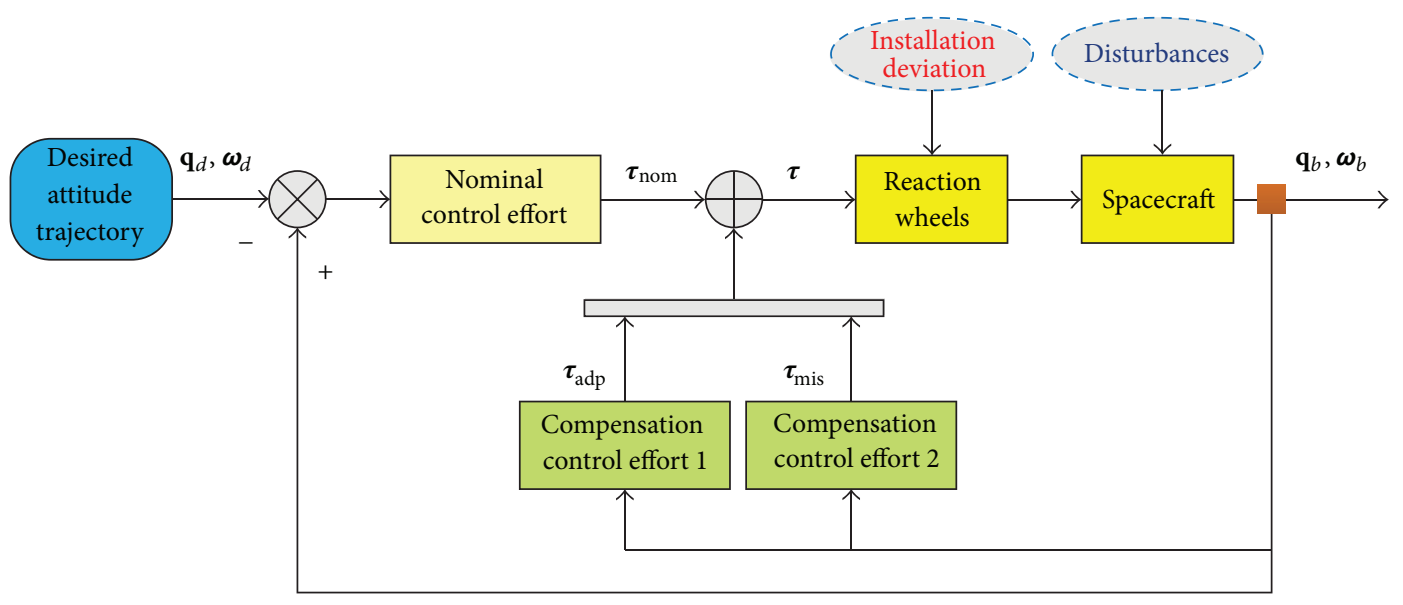

FIGURE 1: Structure of the attitude compensation controller.

critical missions. As a result, more and more investigations also have focused on attitude control design with finite-time convergence. In [21-23], the finite-time control technique was applied to design an attitude controller. Feng et al. [24] proposed a terminal sliding mode controller to solve the singular problem for a second-order nonlinear dynamic system. A terminal sliding mode and the Chebyshev neural network were used in [25] to guarantee that the attitude manoeuvre was accomplished in finite time, even in the presence of an unknown inertia matrix, external disturbances, and control input constraints. Furthermore, two robust sliding mode controllers were proposed in [26] to realize attitude tracking in finite-time. Similar finite-time fault tolerant controllers for spacecrafts were investigated in [27-29].

This work focuses on developing a control scheme to perform attitude compensation for an overactuated spacecraft with reaction wheel installation deviation, external disturbances, and uncertain inertia parameters. More specifically, the attitude tracking error is required to be zero in finite time. The proposed approach is illustrated in Figure 1. The compensation control module is added to the output of the nominal controller to compensate for the reaction wheel misalignment, disturbances, and uncertain moment of inertia. The proposed scheme solves a difficult problem of reliable and high accuracy attitude tracking control in finite time that rejects external disturbances and, at the same time, compensates for actuator misalignment and system uncertainties so that the control objective is met.

The remainder of this paper is organized as follows. In Section 2, we summarize the mathematical model for the rigid spacecraft attitude and control problem. A compensation control solution with the misalignment, disturbance, and mass moment of inertia is presented in Section 3. Simulation results are presented in Section 4. Some conclusions are given in Section 5.

\section{Mathematical Model and Problem Formulation}

The notation adopted throughout this paper is introduced as follows. The symbol $\|\cdot\|$ denotes the standard Euclidean norm or its induced norm; the symbol $\|\cdot\|_{\infty}$ denotes the infinite norm of a vector or matrix. For any given matrix $\mathbf{A} \in \mathbf{R}^{p \times q}$ with full row rank, $\mathbf{A}^{\dagger}$ denotes its pseudoinverse.

2.1. Dynamic Model of Rigid Spacecraft. Consider a rigid space system described by the following attitude kinematics and dynamics equations [30]:

$$
\begin{gathered}
\dot{\mathbf{q}}=\frac{1}{2}\left(\mathbf{q}^{\times}+q_{0} \mathbf{I}_{3}\right) \boldsymbol{\omega}, \\
\dot{q}_{0}=-\frac{1}{2} \mathbf{q}^{T} \boldsymbol{\omega}, \\
\mathbf{J} \dot{\boldsymbol{\omega}}+\boldsymbol{\omega}^{\times} \mathbf{J} \boldsymbol{\omega}=\mathbf{u}+\mathbf{d},
\end{gathered}
$$

where $\boldsymbol{\omega} \in \mathbf{R}^{3}$ is the angular velocity of a body-fixed reference frame expressed in the body-fixed reference frame, $\mathbf{J} \epsilon$ $\mathbf{R}^{3 \times 3}$ (positive and definite) is the total inertia matrix of the spacecraft, $\mathbf{u}=\left[\begin{array}{lll}u_{1} & u_{2} & u_{3}\end{array}\right]^{T} \in \mathbf{R}^{3}$ denotes the combined control torque produced by the actuators, and $\mathbf{d}(t)=$ $\left[\begin{array}{lll}d_{1} & d_{2} & d_{3}\end{array}\right]^{T} \in \mathbf{R}^{3}$ denotes the external disturbance torque from the environment, which is assumed to be unknown but bounded; $q_{0}, \mathbf{q}$ are the scalar and vector components of the unit quaternion, respectively, with $\mathbf{q}=\left[\begin{array}{lll}q_{1} & q_{2} & q_{3}\end{array}\right]^{T} \epsilon$ $\mathbf{R}^{3}$, satisfying the constraint $q_{0}^{2}+\mathbf{q}^{T} \mathbf{q}=1 ; \mathbf{I}_{3}$ represents the identity matrix with proper dimensions, and for $\forall \mathbf{a}=$ $\left[\begin{array}{lll}a_{1} & a_{2} & a_{3}\end{array}\right]^{T}, \mathbf{a}^{\times}$denotes a skew-symmetric matrix, more precisely,

$$
\mathbf{a}^{\times}=\left[\begin{array}{ccc}
0 & -a_{3} & a_{2} \\
a_{3} & 0 & -a_{1} \\
-a_{2} & a_{1} & 0
\end{array}\right]
$$

2.2. Reaction Wheel Configuration with Installation Deviation. For orbiting spacecraft, loosely speaking, they have more than three reaction wheels aligned with the spacecraft body axes. However, in practice, the configuration of actuators will never be perfect; that is, to say, whether due to finite manufacturing tolerances or warping of the spacecraft structure during 
launch, some alignment errors can always exist. Thus, in this section, the reaction wheels misalignment is taken into consideration; the faulty dynamics can be described by

$$
\mathrm{J} \omega=-\omega^{\times} \mathrm{J} \omega+(\mathrm{D}+\Delta \mathrm{D}) \tau+\mathrm{d},
$$

where $\mathbf{D} \in \mathbf{R}^{3 \times N}$ denotes the actuator distribution matrix, $\Delta \mathrm{D}$ denotes the actuator distribution matrix induced by misalignment, and $\boldsymbol{\tau}=\left[\tau_{1}, \ldots, \tau_{N}\right]^{T} \in \mathbf{R}^{N}$ denotes the actual output torque of the $N$ reaction wheels.

Due to the rotation of the payload or the existence of the flywheel installation deviation, the moment of inertia $\mathbf{J}$ is uncertain but positive definite symmetric matrices and record $\mathbf{J}=\mathbf{J}_{0}+\Delta \mathbf{J}$, where $\mathbf{J}_{0}$ denotes the nominal rotational inertia and $\Delta \mathbf{J}$ denotes the uncertain rotational inertia. Here set $0<\|\Delta \mathbf{J}\| \leq\|\mathbf{J}\| \leq J_{\max }<\infty$ and $J_{\max }$ is a positive constant.

2.3. Attitude Tracking Model. Assume that the desired attitude to be followed is described with a desired frame $\mathscr{T}$ with respect to $\mathscr{I}$. It is specified by the desired unit quaternion $\mathbf{Q}_{\mathbf{d}}=\left(q_{d 0}, \mathbf{q}_{\mathbf{d}}^{T}\right) \in \mathbf{R} \times \mathbf{R}^{3}$. The desired angular velocity is denoted by $\boldsymbol{\omega}_{\mathbf{d}} \in \mathbf{R}^{3}$. Let the error quaternion $\mathbf{Q}_{\mathbf{e}}=\left(e_{0}, \mathbf{e}^{T}\right) \epsilon$ $\mathbf{R} \times \mathbf{R}^{3}$ denote the attitude between $\mathscr{B}$ and $\mathscr{T}$, and let $\boldsymbol{\omega}_{\mathrm{e}} \in \mathbf{R}^{3}$ represent the corresponding error angular velocity. One has

$$
\omega_{\mathrm{e}}=\omega-\widetilde{\mathbf{R}} \omega_{\mathrm{d}},
$$

where $\widetilde{\mathbf{R}} \in \mathbf{R}^{3 \times 3}$ denote the corresponding rotation matrix that brings $\mathscr{T}$ onto $\mathscr{B}$, and

$$
\widetilde{\mathbf{R}}=\left(e_{0}^{2}-\mathbf{e}^{T} \mathbf{e}\right) \mathbf{I}_{3}+2 \mathbf{e} \mathbf{e}^{T}-2 e_{0} \mathbf{e}^{\times} .
$$
by:

With (1)-(5), the attitude tracking error dynamics is given

$$
\begin{gathered}
\mathbf{J} \dot{\omega}_{\mathbf{e}}+\left(\dot{\boldsymbol{\omega}}_{\mathrm{e}}+\widetilde{\mathbf{R}} \boldsymbol{\omega}_{\mathbf{d}}\right)^{\times} \mathbf{J}\left(\boldsymbol{\omega}_{\mathbf{e}}+\widetilde{\mathbf{R}} \boldsymbol{\omega}_{\mathbf{d}}\right)-\mathbf{J}\left(\boldsymbol{\omega}_{\mathbf{e}}^{\times} \widetilde{\mathbf{R}} \boldsymbol{\omega}_{\mathbf{d}}-\widetilde{\mathbf{R}} \boldsymbol{\omega}_{\mathbf{d}}\right) \\
=(\mathbf{D}+\Delta \mathbf{D}) \mathbf{E}(t) \boldsymbol{\tau}(t)+\mathbf{d}(t), \\
\dot{e}_{0}=-\frac{1}{2} \mathbf{e}^{T} \boldsymbol{\omega}_{\mathrm{e}}, \\
\dot{\mathbf{e}}=\frac{1}{2}\left(\mathbf{e}^{\times}+e_{0} \mathbf{I}_{3}\right) \boldsymbol{\omega}_{\mathbf{e}} .
\end{gathered}
$$

2.4. Control Objective. The control objective of this work can be stated as considering the uncertain attitude tracking system (8)-(10) and design a control law to guarantee that the attitude tracking error converges to zero in finite-time, even in the presence of actuator misalignment, uncertain inertia matrix, and external disturbance $\mathbf{d}(t)$.

We present now the main results of this study.

\section{Finite-Time Attitude Compensation Control}

For the proposed control approach shown in Figure 1, the nominal control power and the compensation control effort are presented in this section. First, a finite-time sliding mode surface is proposed. Then, based on the finite-time sliding mode surface, a compensation controller is synthesized and added to the nominal controller to guarantee the global asymptotic stability of the resulting closed-loop attitude tracking system with finite-time convergence.

3.1. Finite-Time Sliding Mode Surface Design. We first introduce some lemmas which will be utilized in the subsequent control development and analysis.

Lemma 1 (see [31]). If $p \in(0,1)$, then the following inequality holds for any vector $\mathbf{x}=\left(x_{1}, x_{2}, \ldots, x_{n}\right)^{T} \in \mathbf{R}^{n}$ :

$$
\sum_{i=1}^{n}\left|x_{i}\right|^{1+p} \geq\left(\sum_{i=1}^{n}\left|x_{i}\right|^{2}\right)^{(1+p) / 2}
$$

Lemma 2 (see [32]). Suppose that $\mathbf{V}(\mathbf{x})$ is a $C^{1}$ smooth positive-definite function such that

$$
\dot{\mathbf{V}}(\mathbf{x})+\lambda_{1} \mathbf{V}(\mathbf{x})+\lambda_{2} \mathbf{V}^{\beta}(\mathbf{x}) \leq 0
$$

where $\lambda_{1} \in \mathbf{R}^{+}, \lambda_{2} \in \mathbf{R}^{+}, \beta \in \mathbf{R}^{+}$and $0<\beta<1$. Then for any initial value $x(0)=x_{0}$, it follows that $\mathbf{V}(\mathbf{x}(t))=0$ for all the time $t \geq t_{F_{1}}$,

$$
t_{F_{1}} \leq \frac{1}{\lambda_{1}(1-\beta)} \ln \frac{\lambda_{1} \mathbf{V}^{1-\beta}\left(x_{0}\right)+\lambda_{2}}{\lambda_{2}} .
$$

To this end, in this work, a sliding mode surface is introduced as

$$
\mathbf{s}=\boldsymbol{\omega}_{\mathbf{e}}+\mu_{1} \mathbf{e}+\mu_{2} \operatorname{sgn}(\mathbf{e})^{r},
$$

where $\mathbf{s}=\left[\begin{array}{lll}s_{1} & s_{2} & s_{3}\end{array}\right]^{T} \in \mathbf{R}^{3}, \mu_{1}>0, \mu_{2}>0$, and $0<\gamma<1$ are the design parameters and $\operatorname{sgn}(\mathbf{e})$ is the sign function defined by

$$
\operatorname{sgn}(\mathbf{e})^{r}=\left(\left|e_{1}\right|^{r} \operatorname{sgn}\left(e_{1}\right),\left|e_{2}\right|^{r} \operatorname{sgn}\left(e_{2}\right),\left|e_{3}\right|^{r} \operatorname{sgn}\left(e_{3}\right)\right)^{T} \text {. }
$$

Theorem 3. If an controller $\mathbf{u}(t)$ is appropriately designed to let the states reach the sliding surface $\mathbf{s}$, then it has $\mathbf{e}(t) \equiv 0$, $e_{0}(t) \equiv 1$, and $\omega_{\mathbf{e}}(t) \equiv 0$ for all the $t \geq t_{F 1}$.

Proof. From the sliding mode theory [33], it is known that once the state trajectories of the attitude tracking system reach the sliding surface, that is, $\mathbf{s}=\mathbf{0}$, it follows that

$$
\boldsymbol{\omega}_{\mathbf{e}}=-\mu_{1} \mathbf{e}-\mu_{2} \operatorname{sgn}(\mathbf{e})^{r} .
$$

Consider a candidate Lyapunov function as

$$
V_{1}=\left(1-e_{0}\right)^{2}+\mathbf{e}^{T} \mathbf{e} .
$$

Because the inequality $\sum_{i=0}^{3}\left|e_{i}\right|^{r+1} \geq\left(\sum_{i=0}^{3}\left|e_{i}\right|^{2}\right)^{(r+1) / 2}$ holds for $0<r<1$, it is obtained from (15) that

$$
\dot{V}_{1}=-2 \dot{e}_{0}=\mathbf{e}^{T} \boldsymbol{\omega}_{\mathbf{e}}=-\mu_{1} \mathbf{e}^{T} \mathbf{e}-\mu_{2} \sum_{i=1}^{3}\left|e_{i}\right|^{r+1}
$$


which implies that $\dot{V}_{1}=0$ if and only if $\mathbf{e}=0$. Thus, $V_{1}$ is really a Lyapunov function such that the signal e will converge to zero and, accordingly, $e_{0}$ tends to \pm 1 as $t \rightarrow \infty$ by using the constraint in (18). Note that the equilibrium point $\left(e_{0}, \mathbf{e}\right)=(-1, \mathbf{0})$ is not a stable equilibrium point [34]. Then, by Lemma 1 , we obtain

$$
\dot{V}_{1} \leq-\mu_{1} \mathbf{e}^{T} \mathbf{e}-\mu_{2}\left(\mathbf{e}^{T} \mathbf{e}\right)^{(r+1) / 2} .
$$

Because $\mathbf{e}=(-1,0)^{T}$ is not the stable equilibrium point, the signal e will converge to zero. Thus, $\lim _{t \rightarrow \infty} e_{0}(t)=1$ can be obtained from the constraint $\mathbf{e}^{T} \mathbf{e}+e_{0}^{2}=1$. There exists a finite time $\bar{t} \geq 0$ such that $e_{0}(t)>0$ for $t \geq \bar{t}$. Then, for $t \geq \bar{t}$, one has

$$
\begin{aligned}
\left(1-e_{0}\right)^{2} & =2 e_{0}^{2}+\mathbf{e}^{T} \mathbf{e}-2 e_{0} \\
& =2 e_{0}\left(e_{0}-1\right)+\mathbf{e}^{T} \mathbf{e}-2 \leq \mathbf{e}^{T} \mathbf{e} .
\end{aligned}
$$

Then,

$$
V_{1} \leq 2 \mathbf{e}^{T} \mathbf{e}
$$

Using (21), (19) can be further bounded by

$$
\dot{V}_{1} \leq-\frac{1}{2} \mu_{1} V_{1}-\left(\frac{1}{2}\right)^{(r+1) / 2} \mu_{2} V_{1}^{(r+1) / 2} .
$$

Using $0.5<(r+1) / 2<1$ and Lemma 2 , one has $V_{1}(t) \equiv 0$ for all $t \geq t_{F 1}$. According to definition of $V_{1}(t)$ in (17), $e_{0}(t) \equiv$ $1, \mathbf{e}(t) \equiv 0$, and $\boldsymbol{\omega}_{\mathbf{e}}(t) \equiv 0$ for all $t \geq t_{F 1}$ are concluded. Thereby, the proof is completed here.

3.2. Attitude Compensation Controller Design. Considering the reaction wheel installation deviation and external disturbance, it is obtained from the sliding surface (14) that

$$
\mathbf{J} \dot{\mathbf{s}}=\mathbf{D} \boldsymbol{\tau}+\Delta \mathbf{D} \boldsymbol{\tau}+\mathbf{L}-\beta \mathbf{q}_{e}-0.5 \mathbf{J} \mathbf{s}
$$

Because $\mathbf{J}$ is unknown but bounded, then $\mathbf{J}=0$ is established. Then, $\mathbf{L}$ can be represented to be bounded by $\|\mathbf{L}\| \leq \alpha_{0}+\alpha_{1}\|\boldsymbol{\omega}\|+\alpha_{2}\|\boldsymbol{\omega}\|^{2}$, where $\boldsymbol{\alpha}_{i}, i=1,2,3$ are positive constants [35].

In order to facilitate analysis and proof, firstly, define $\kappa=\lambda_{\min }\left(\mathbf{D} \mathbf{D}^{T}\right), 3\|\Delta \mathbf{D}\|_{\infty}\left\|\mathbf{D}^{\dagger}\right\|_{\infty}=\varepsilon<1$, and $\mathbf{D}^{\dagger}$ is the pseudoinverse of $\mathbf{D}$. Now, we are ready to present the main result in Theorem 4 .

Theorem 4. Considering the uncertainty attitude tracking dynamics described by (5) with actuator misalignment $\Delta \mathbf{D}$ and external disturbance torque $\mathbf{d}(t)$, design an attitude compensation control law as

$$
\boldsymbol{\tau}=\boldsymbol{\tau}_{\text {nom }}(t)+\boldsymbol{\tau}_{a d p}(t)+\boldsymbol{\tau}_{\text {mis }}(t),
$$

where

$$
\begin{gathered}
\boldsymbol{\tau}_{\text {nom }}(t)=-k_{1} \beta \frac{\mathbf{D}^{T}\left\|\mathbf{q}_{e}\right\| \mathbf{s}}{\|\mathbf{s}\|}-K \frac{\mathbf{D}^{T} \mathbf{s}}{\|\mathbf{s}\|^{2}}, \\
\boldsymbol{\tau}_{\text {adp }}(t)=\frac{\mathbf{D}^{T}\left(-\widehat{k}_{3}-\widehat{k}_{4}\|\boldsymbol{\omega}\|-\widehat{k}_{5}\|\boldsymbol{\omega}\|^{2}\right) \mathbf{s}}{\|\mathbf{s}\|}, \\
\boldsymbol{\tau}_{\text {mis }}(t)=-\frac{\left(\widehat{\pi}_{1}-1\right) \varphi(t) \mathbf{D}^{\dagger} \mathbf{s}}{\|\mathbf{s}\|_{\infty}}
\end{gathered}
$$

where $K \in \mathbf{R}^{+}$is control parameter and $k_{1}$ is carefully chosen such that $k_{1} \kappa-1>0 ; \widehat{\pi}_{1}$ is the estimate of $\pi_{1}=1 /(1-\delta) ; \widehat{k}_{3}$ is the estimate of $k_{3}=\alpha_{0} / \kappa ; \widehat{k}_{4}$ is the estimate of $k_{4}=\alpha_{1} / \kappa$; $\widehat{k}_{5}$ is the estimate of $k_{5}=\alpha_{2} / \kappa$. Moreover, $\dot{\hat{\pi}}_{1}, \widehat{k}_{i}, i=3,4,5$ are adaptively updated by $\dot{\hat{k}}_{2}=\left(\left\|\mathbf{s}^{T}\right\| / \ell_{4}\right) \widehat{k}_{i}, \dot{\hat{k}}_{3}=\|\boldsymbol{\omega}\|\|\mathbf{s}\| / \ell_{5}$, $\dot{\hat{k}}_{4}=\|\boldsymbol{\omega}\|^{2}\|\mathbf{s}\| / \ell_{6}$, and $\dot{\vec{\pi}}_{1}=l_{1} \varphi(t)\|\mathbf{s}\|_{\infty}$, respectively. Then the system states reach the sliding mode surface $\mathbf{s}(t)=0$ in finitetime for any initial state $\mathbf{Q}(0)$ and $\boldsymbol{\omega}(0)$.

Proof. When $\mathbf{s} \neq \mathbf{0}$, consider a candidate Lyapunov function:

$$
V_{2}=\frac{1}{2} \mathbf{s}^{T} \mathbf{J} \mathbf{s}+\frac{(1-\varepsilon)}{2 l_{1}} \tilde{\pi}_{1}^{2}+\frac{1}{2} l_{3} \widetilde{k}_{3}^{2}+\frac{1}{2} l_{4} \widetilde{k}_{4}^{2}+\frac{1}{2} l_{5} \widetilde{k}_{5}^{2},
$$

where $\tilde{\pi}_{1}=\pi_{1}-\widehat{\pi}_{1}, \tilde{k}_{i}=k_{i}-\widehat{k}_{i}, i=3,4,5$, and $l_{1}, l_{3}, l_{4}, l_{5}$ are the positive constants.

Calculating the time-derivative of $V_{2}$, it yields

$$
\begin{aligned}
& \dot{V}_{2} \leq \mathbf{s}^{T} \mathbf{J} \dot{\mathbf{s}}-\frac{(1-\varepsilon)}{l_{1}} \tilde{\pi} \dot{\hat{\pi}}-l_{3} \widetilde{k}_{3} \dot{\widehat{k}}_{3}-l_{4} \widetilde{k}_{4} \dot{\widehat{\hat{k}}}_{4}-l_{5} \dot{\widehat{\hat{k}}}_{3} \\
& =\mathbf{s}\left(\mathbf{D} \boldsymbol{\tau}_{\mathrm{nom}}-\beta \mathbf{q}_{e}\right)+\mathbf{s}\left(\mathbf{D} \boldsymbol{\tau}_{\mathrm{adp}}+\mathbf{L}\right)+\mathbf{s}\left(\mathbf{D} \boldsymbol{\tau}_{\text {mis }}+\Delta \mathbf{D} \boldsymbol{\tau}\right) \\
& -\frac{(1-\varepsilon)}{l_{1}} \tilde{\pi} \dot{\hat{\pi}}-l_{3} \widetilde{k}_{3} \dot{\widehat{k}}_{3}-l_{4} \widetilde{k}_{4} \dot{\widehat{\hat{k}}}_{4}-l_{5} \dot{\widehat{\hat{k}}}_{3} .
\end{aligned}
$$

With (25), it follows that

$$
\begin{aligned}
\mathbf{s}\left(\mathbf{D} \boldsymbol{\tau}_{\text {nom }}-\beta \mathbf{q}_{e}\right) & \leq \mathbf{s} \mathbf{D} \boldsymbol{\tau}_{\text {nom }}+\beta\left\|\mathbf{q}_{e}\right\|\|\mathbf{s}\| \\
& \leq-\kappa k_{1} \beta\|\mathbf{s}\|\left\|\mathbf{q}_{e}\right\|+\beta\left\|\mathbf{q}_{e}\right\|\|\mathbf{s}\|-K \kappa \\
& =-\left(k_{1} \kappa \beta-\beta\right)\left\|\mathbf{q}_{e}\right\|-\kappa K \\
& \leq-\kappa K .
\end{aligned}
$$

In the same way, with (26), the following equality is yielded:

$$
\begin{aligned}
\mathbf{s}\left(\mathbf{D} \boldsymbol{\tau}_{\text {adp }}+\mathbf{L}\right) \leq & \mathbf{s} \mathbf{D} \boldsymbol{\tau}_{\text {adp }}+\|\mathbf{s}\|\|\mathbf{L}\| \\
\leq & -\kappa\left(\widehat{k}_{3}-\frac{\alpha_{0}}{\kappa}\right)\|\mathbf{s}\|-\kappa\left(\widehat{k}_{4}-\frac{\alpha_{1}}{\kappa}\right)\|\mathbf{s}\|\|\boldsymbol{\omega}\| \\
& -\kappa\left(\widehat{k}_{5}-\frac{\alpha_{2}}{\kappa}\right)\|\mathbf{s}\|\|\boldsymbol{\omega}\|^{2} \\
= & \kappa \widetilde{k}_{3}\|\mathbf{s}\|+\kappa \widetilde{k}_{4}\|\mathbf{s}\|\|\boldsymbol{\omega}\|+\kappa \widetilde{k}_{5}\|\mathbf{s}\|\|\boldsymbol{\omega}\|^{2} .
\end{aligned}
$$


Define $\varphi(t)=\left\|\mathbf{D}^{\dagger}\left(\boldsymbol{\tau}_{\text {nom }}+\boldsymbol{\tau}_{\text {adp }}\right)\right\|_{\infty}$. If the choice of the control gains is such that $3\|\Delta \mathbf{D}\|_{\infty}\left\|\mathbf{D}^{-1}\right\|_{\infty}=\varepsilon<1$, using the inequality $\mathbf{x y} \leq 3\|\mathbf{x}\|_{\infty}\|\mathbf{y}\|_{\infty}>0$ for any vector $\mathbf{x}, \mathbf{y} \in \mathbf{R}^{n}$ and applying (29) lead to

$$
\begin{aligned}
& \mathbf{s}\left(\mathbf{D} \boldsymbol{\tau}_{\text {mis }}+\Delta \mathbf{D} \boldsymbol{\tau}\right) \\
&=\mathbf{s} \Delta \mathbf{D}\left(\boldsymbol{\tau}_{\text {nom }}+\boldsymbol{\tau}_{\text {adp }}+\boldsymbol{\tau}_{\text {mis }}\right)+\mathbf{s} \mathbf{D} \boldsymbol{\tau}_{\text {mis }} \\
& \leq 3\|\Delta \mathbf{D}\|_{\infty}\left\|\mathbf{D}^{\dagger}\right\|_{\infty} \varphi(t)\|\mathbf{s}\|_{\infty}+\mathbf{s} \Delta \mathbf{D} \boldsymbol{\tau}_{\text {mis }}+\mathbf{s} \mathbf{D} \boldsymbol{\tau}_{\text {mis }} \\
& \leq \varepsilon \varphi(t)\|\mathbf{s}\|_{\infty}-\left(\widehat{\pi}_{1}-1\right) \varphi(t)\|\mathbf{s}\|_{\infty} \\
&+\mathbf{s} \Delta \mathbf{D}\left[-\frac{\left(\widehat{\pi}_{1}-1\right) \varphi(t) \mathbf{D}^{\dagger} \mathbf{s}}{\|\mathbf{s}\|_{\infty}}\right] \\
&=(1-\varepsilon) \widetilde{\pi}_{1} \varphi(t)\|\mathbf{s}\|_{\infty} .
\end{aligned}
$$
that

Substituting (30)-(32) into (29), consequently, it follows

$$
\dot{V} \leq-\kappa K \text {. }
$$

And then,

$$
\begin{aligned}
& \int_{0}^{t} \dot{V}_{2}(\mu) d \mu \leq-\kappa K \int_{0}^{t} d \mu ; \text { that is, } \\
& V_{2}(t)-V_{2}(0) \leq-\kappa K t .
\end{aligned}
$$

Due to $V_{2}(t) \geq 0$, solving (34) leads to $V_{2}(t) \equiv 0$, for $t \geq$ $t_{F 2}$,

$$
t_{F 2} \leq-\frac{V_{2}(t)-V_{2}(0)}{\kappa K} .
$$

Then, it can be concluded that the system states reach the surface $\mathbf{s}(t)=\mathbf{0}$ in finite time. Thereby, the proof is completed here.

Remark 5. The controller (24) includes three parts: $\boldsymbol{\tau}_{\text {adp }}(t)$ is used to compensate for system uncertainty caused by the external disturbance and moment inertia, $\boldsymbol{\tau}_{\text {mis }}(t)$ is used to accommodate actuator misalignment, and $\boldsymbol{\tau}_{\text {nom }}(t)$ is the nominal control.

Theorem 6. Consider the attitude tracking system given by (5), (9), and (10). If the control scheme (24) is implemented, then the attitude tracking maneuver can be accomplished in a finite time $t_{F}=t_{F 1}+t_{F 2}$; that is, $\boldsymbol{\omega}_{\mathbf{e}}(t) \equiv 0$ and $\mathbf{e}(t) \equiv 0$ are guaranteed for all the time $t \geq t_{F}$.

Proof. It is obtained from Theorem 4 that all the states of the attitude tracking system reach the sliding mode surface $\mathbf{s}(t)=0$ in finite-time $t_{F 2}$ and maintain the motion state on the slide mode surface. Furthermore, from Theorem 3, it is obtained that once the system state reaches the slide mode surface (14) the system state can reach the equilibrium point $\left(e_{0}, \mathbf{e}\right)=(1,0)$ in finite time $t_{F 1}$. Therefore, for any initial state $\mathbf{Q}(0)$ and $\boldsymbol{\omega}(0)$, the desired attitude trajectory can be followed in a finite time $t_{F}$; that is, $\mathbf{e}(t) \equiv 0, e_{0}(t) \equiv 1$, and $\omega_{\mathbf{e}}(t) \equiv 0$ are achieved for all the time $t \geq t_{F}$. Thereby, the proof is completed here.

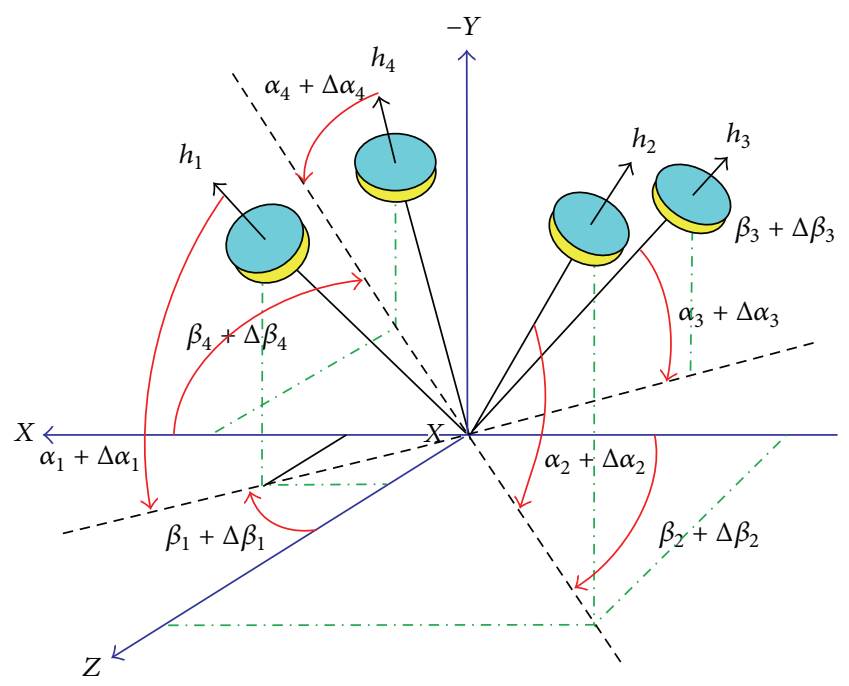

Figure 2: Configuration of four reaction wheels.

\section{Numerical Simulation Results}

4.1. Reaction Wheel Configuration. To demonstrate the effectiveness and performance of the proposed compensation control scheme, numerical simulations have been carried out using the rigid spacecraft system (3) and (6) in conjunction with the developed compensation control law (24). The spacecraft is activated by four reaction wheels with a limited control torque $u_{\max }=0.1 \mathrm{~N} \cdot \mathrm{m}$. The configuration of those four actuators is shown in Figure 2. $\boldsymbol{\alpha}_{i}=35.26^{\circ}$ and $\boldsymbol{\beta}_{i}=45^{\circ}$ are the nominal alignment angles, $i=1,2,3,4 . \Delta \boldsymbol{\alpha}_{i}$ and $\Delta \boldsymbol{\beta}_{i}$ are the misalignment angles.

With the configuration shown in Figure 2, the relation between the actual output torque of reaction wheel and the total torque acting on the spacecraft is to be calculated as

$$
\begin{aligned}
\mathbf{u}(t)= & \tau_{1}\left(\begin{array}{c}
\cos \left(\alpha_{1}+\Delta \alpha_{1}\right) \sin \left(\beta_{1}+\Delta \beta_{1}\right) \\
-\sin \left(\alpha_{1}+\Delta \alpha_{1}\right) \\
\cos \left(\alpha_{1}+\Delta \alpha_{1}\right) \cos \left(\beta_{1}+\Delta \beta_{1}\right)
\end{array}\right) \\
& +\tau_{2}\left(\begin{array}{c}
-\cos \left(\alpha_{2}+\Delta \alpha_{2}\right) \cos \left(\beta_{2}+\Delta \beta_{2}\right) \\
-\sin \left(\alpha_{2}+\Delta \alpha_{2}\right) \\
\cos \left(\alpha_{2}+\Delta \alpha_{2}\right) \sin \left(\beta_{2}+\Delta \beta_{2}\right)
\end{array}\right) \\
& +\tau_{3}\left(\begin{array}{c}
-\cos \left(\alpha_{3}+\Delta \alpha_{3}\right) \sin \left(\beta_{3}+\Delta \beta_{3}\right) \\
-\sin \left(\alpha_{3}+\Delta \alpha_{3}\right) \\
-\cos \left(\alpha_{3}+\Delta \alpha_{3}\right) \cos \left(\beta_{3}+\Delta \beta_{3}\right)
\end{array}\right) \\
& +\tau_{4}\left(\begin{array}{c}
\cos \left(\alpha_{4}+\Delta \alpha_{4}\right) \cos \left(\beta_{4}+\Delta \beta_{4}\right) \\
-\sin \left(\alpha_{4}+\Delta \alpha_{4}\right) \\
-\cos \left(\alpha_{4}+\Delta \alpha_{4}\right) \sin \left(\beta_{4}+\Delta \beta_{4}\right)
\end{array}\right) .
\end{aligned}
$$

Although the misalignment angles exist due to finitemanufacture technique and vehicle vibration, those angles 
$\Delta \boldsymbol{\alpha}_{i}, \Delta \boldsymbol{\beta}_{i}(i=1,2,3,4)$ are small values. They can be approximated by

$$
\begin{aligned}
& \cos \Delta \boldsymbol{\alpha}_{i} \approx 1, \quad \sin \Delta \boldsymbol{\alpha}_{i} \approx \Delta \boldsymbol{\alpha}_{i}, \quad \cos \Delta \boldsymbol{\beta}_{i} \approx 1, \\
& \sin \Delta \boldsymbol{\beta}_{i} \approx \Delta \boldsymbol{\beta}_{i}, \quad \sin \Delta \boldsymbol{\alpha}_{i} \sin \Delta \boldsymbol{\beta}_{i} \approx 0
\end{aligned}
$$

$$
(i=1,2,3,4) \text {. }
$$

Hence, (36) can be re written as

$$
\mathbf{u}(t)=\mathbf{D} \boldsymbol{\tau}(t)+\Delta \mathbf{D} \boldsymbol{\tau}(t)
$$

where $\mathbf{D}$ and $\Delta \mathbf{D}=\left(\Delta \mathbf{D}_{1}, \Delta \mathbf{D}_{2}, \Delta \mathbf{D}_{3}, \Delta \mathbf{D}_{4}\right)$ are calculated as

$$
\begin{aligned}
& \mathbf{D}=\left(\begin{array}{cccc}
\cos \alpha_{1} \sin \beta_{1} & -\cos \alpha_{2} \sin \beta_{2} & -\cos \alpha_{3} \sin \beta_{3} & \cos \alpha_{4} \sin \beta_{4} \\
-\sin \alpha_{1} & -\sin \alpha_{2} & -\sin \alpha_{3} & -\sin \alpha_{4} \\
\cos \alpha_{1} \cos \beta_{1} & \cos \alpha_{2} \cos \beta_{2} & -\cos \alpha_{3} \cos \beta_{3} & -\cos \alpha_{4} \cos \beta_{4}
\end{array}\right) \\
& =\left(\begin{array}{cccc}
\frac{\sqrt{3}}{3} & -\frac{\sqrt{3}}{3} & -\frac{\sqrt{3}}{3} & \frac{\sqrt{3}}{3} \\
-\frac{\sqrt{3}}{3} & -\frac{\sqrt{3}}{3} & -\frac{\sqrt{3}}{3} & -\frac{\sqrt{3}}{3} \\
\frac{\sqrt{3}}{3} & \frac{\sqrt{3}}{3} & -\frac{\sqrt{3}}{3} & -\frac{\sqrt{3}}{3}
\end{array}\right), \\
& \Delta \mathbf{D}_{1}=\left(\begin{array}{c}
\Delta \beta_{1} \cos \alpha_{1} \cos \beta_{1}-\Delta \alpha_{1} \sin \alpha_{1} \sin \beta_{1} \\
-\Delta \alpha_{1} \cos \alpha_{1} \\
-\Delta \beta_{1} \cos \alpha_{1} \sin \beta_{1}-\Delta \beta_{1} \sin \alpha_{1} \cos \beta_{1}
\end{array}\right) \\
& \Delta \mathbf{D}_{2}=\left(\begin{array}{c}
-\Delta \beta_{2} \cos \alpha_{2} \cos \beta_{2}+\Delta \alpha_{2} \sin \alpha_{2} \sin \beta_{2} \\
-\Delta \alpha_{2} \cos \alpha_{2} \\
-\Delta \beta_{2} \cos \alpha_{2} \sin \beta_{2}-\Delta \beta_{2} \sin \alpha_{2} \cos \beta_{2}
\end{array}\right) \\
& \Delta \mathbf{D}_{3}=\left(\begin{array}{c}
-\Delta \beta_{3} \cos \alpha_{3} \cos \beta_{3}+\Delta \alpha_{3} \sin \alpha_{3} \sin \beta_{3} \\
-\Delta \alpha_{3} \cos \alpha_{3} \\
\Delta \beta_{3} \cos \alpha_{3} \sin \beta_{3}+\Delta \beta_{3} \sin \alpha_{3} \cos \beta_{3}
\end{array}\right) \\
& \Delta \mathbf{D}_{4}=\left(\begin{array}{c}
\Delta \beta_{4} \cos \alpha_{4} \cos \beta_{4}-\Delta \alpha_{4} \sin \alpha_{4} \sin \beta_{4} \\
-\Delta \alpha_{4} \cos \alpha_{4} \\
\Delta \beta_{4} \cos \alpha_{4} \sin \beta_{4}+\Delta \alpha_{4} \sin \alpha_{4} \cos \beta_{4}
\end{array}\right) \text {. }
\end{aligned}
$$

Remark 7. Theorem 4 gives out the sufficient condition $3\|\Delta \mathbf{D}\|_{\infty}\left\|\mathbf{D}^{\dagger}\right\|_{\infty}=\varepsilon<1$ of efficacious processes on reaction wheel installation deviation $\Delta \mathbf{D}$ for guaranteeing the attitude controller (24). Particulary, according to the definition $\Delta \mathbf{D}$ and matrix norm, then $\|\Delta \mathbf{D}\|_{\infty} \leq \max _{i=1,2,3} \theta_{i}$, where

$$
\begin{aligned}
\theta_{1}= & \sum_{i=1}^{4}\left|\Delta \boldsymbol{\alpha}_{i} \sin \boldsymbol{\alpha}_{i} \sin \boldsymbol{\beta}_{i}\right|+\sum_{i=1}^{4}\left|\Delta \boldsymbol{\beta}_{i} \cos \boldsymbol{\alpha}_{i} \cos \boldsymbol{\beta}_{i}\right| \\
& =\frac{\sqrt{6}}{6} \sum_{i=1}^{4}\left|\Delta \boldsymbol{\alpha}_{i}\right|+\frac{\sqrt{3}}{3} \sum_{i=1}^{4}\left|\Delta \boldsymbol{\beta}_{i}\right|, \\
\theta_{2}= & \sum_{i=1}^{4}\left|\Delta \boldsymbol{\alpha}_{i} \cos \boldsymbol{\alpha}_{i}\right|=\frac{\sqrt{6}}{3} \sum_{i=1}^{4}\left|\Delta \boldsymbol{\alpha}_{i}\right|, \\
\theta_{3}= & \sum_{i=1}^{4}\left|\Delta \boldsymbol{\alpha}_{i} \sin \boldsymbol{\alpha}_{i} \cos \boldsymbol{\beta}_{i}\right|+\sum_{i=1}^{4}\left|\Delta \boldsymbol{\beta}_{i} \cos \boldsymbol{\alpha}_{i} \sin \boldsymbol{\beta}_{i}\right| \\
& =\frac{\sqrt{6}}{6} \sum_{i=1}^{4}\left|\Delta \boldsymbol{\alpha}_{i}\right|+\frac{\sqrt{3}}{3} \sum_{i=1}^{4}\left|\Delta \boldsymbol{\beta}_{i}\right| .
\end{aligned}
$$

On the other hand, inequality $3\|\Delta \mathbf{D}\|_{\infty}\left\|\mathbf{D}^{\dagger}\right\|_{\infty}=\varepsilon<1$ means that $\|\Delta \mathbf{D}\|_{\infty}=\varepsilon /\left(3\left\|\mathbf{D}^{\dagger}\right\|_{\infty}\right)<1 /\left(3\left\|\mathbf{D}^{\dagger}\right\|_{\infty}\right)$. Therefore, the establishment conditions $\max _{i=1,2,3,4} \theta_{i}<1 / 3\left\|\mathbf{D}^{\dagger}\right\|_{\infty}=$ $0.2566 \mathrm{rad}$ of Theorem 4 from (40) can be obtained, that is to say, the installation deviation angle of any two reaction wheels is not larger than $0.2566 \mathrm{rad}$. Relying on this, this largest installation deviation angle, that is $\max _{i=1,2,3,4} \theta_{i}=14.7021^{\circ}$, of the reaction wheel installation structure is considered in this paper.

4.2. Simulation Results. The nominal inertia matrix of the considered spacecraft is specified by [36]

$$
\begin{gathered}
\mathbf{J}_{0}=\left(\begin{array}{ccc}
35 & 3 & -1.5 \\
3 & 28 & 2 \\
-1.5 & 2 & 30
\end{array}\right) \mathrm{kg} \cdot \mathrm{m}^{2}, \\
\Delta \mathbf{J}=\left(1+e^{-0.1 t}+2 \vartheta(t-10)-4 \vartheta(t-20)\right) \operatorname{diag}(3,2,1)
\end{gathered}
$$

which incorporated into the model, where $\vartheta(\cdot)$ is defined as $\vartheta(t \geq 0)=1$ and $\vartheta(t<0)=0$. External disturbance $\mathbf{d}(t)$ is chosen as [35]

$$
\mathbf{d}(t)=\left(\|\boldsymbol{\omega}\|^{2}+0.05\right)(\sin 0.8 t, \cos 0.5 t, \cos 0.3 t)^{T} .
$$

The reaction wheel misalignment angle $\Delta \boldsymbol{\alpha}_{i}(i=1,2,3,4)$ can be selected randomly between $-4.5^{\circ} \sim+4.5^{\circ}$, and $\Delta \boldsymbol{\beta}_{i}(i=$ $1,2,3,4)$ can be selected randomly between $-5.5^{\circ} \sim+5.5^{\circ}$.

In this simulation, spacecraft initial parameter is set as follows: initial angular velocity $\boldsymbol{\omega}_{i}(0)=$ $\left[\begin{array}{lll}0.1 & -0.1-0.05\end{array}\right]^{\circ} / s, i=1,2,3$; initial attitude quaternary $\mathbf{q}_{i}(0)=\left[\begin{array}{llll}0.181 & -0.287 & 0.792 & -0.524\end{array}\right]^{T}, i=0,1,2,3$; the corresponding initial roll angle, pitch angle, and yaw angle are set $0.2^{\circ},-0.4^{\circ}$ and $-0.3^{\circ}$ respectively. The control gains are selected by the following list: $\beta=0.7, K=0.05, \kappa=1.05$, $k_{i}=1.5(i=1,3,4,5)$, as $l_{i}=1.5(i=1,3,4,5)$; moreover, the initial of the adaptive update laws are $\dot{\vec{\pi}}_{1}(0)=1.25$, $\dot{\hat{k}}_{3}(0)=0.68, \dot{\hat{k}}_{4}(0)=0.42, \dot{\hat{k}}_{5}(0)=0.22$.

To demonstrate the effectiveness of the proposed misalignment compensation and disturbance rejection scheme, a spacecraft is numerically simulated using the proposed control compensation strategy (24).

We see in Figures 3-8 the controller managed to stabilize the origin equilibrium point in 30 seconds with great pointing accuracy. Indeed, since the knowledge of spacecraft inertia parameters was not required and an implicit integral item was incorporated in the control law design, external disturbance effect on the attitude control performance can be compensated efficiently, and also great robustness to system uncertainties, such as misalignment, can be guaranteed.

We can see in Figures 3 and 4 the time responses of angle velocity and attitude angle; the proposed control scheme surely realized the high precision stable control in the presence of external disturbance, uncertain moment of inertia, and reaction wheel misalignment, and the pointing accuracy is superior to $0.01^{\circ}$; the attitude stable precision is superior to $0.001^{\circ} / \mathrm{s}$. Meanwhile, from Figures 5 and 6 , 


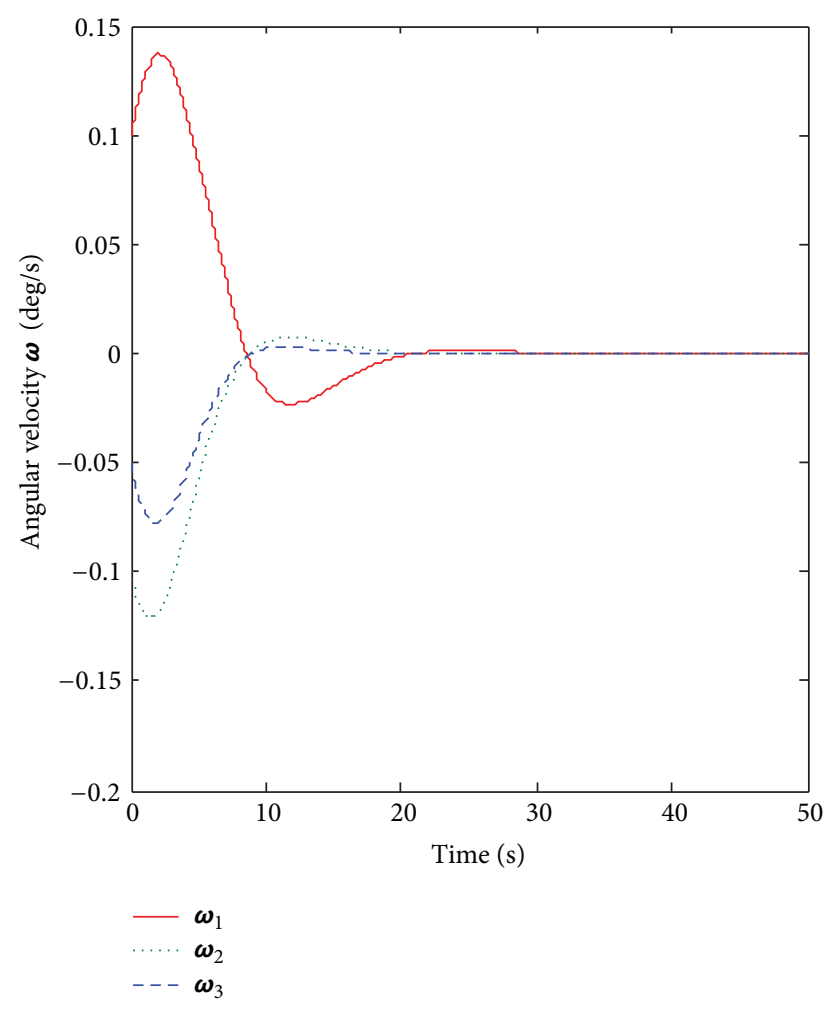

Figure 3: Time responses of angular velocity.

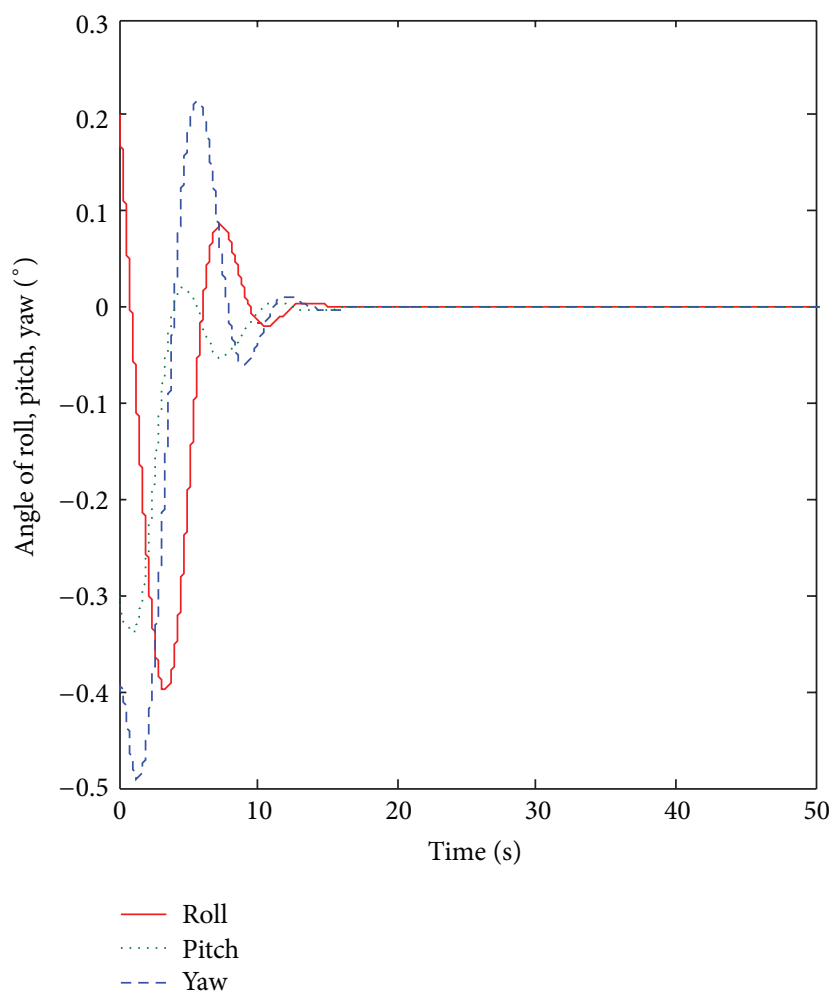

Figure 4: Time response of satellite attitude angle.

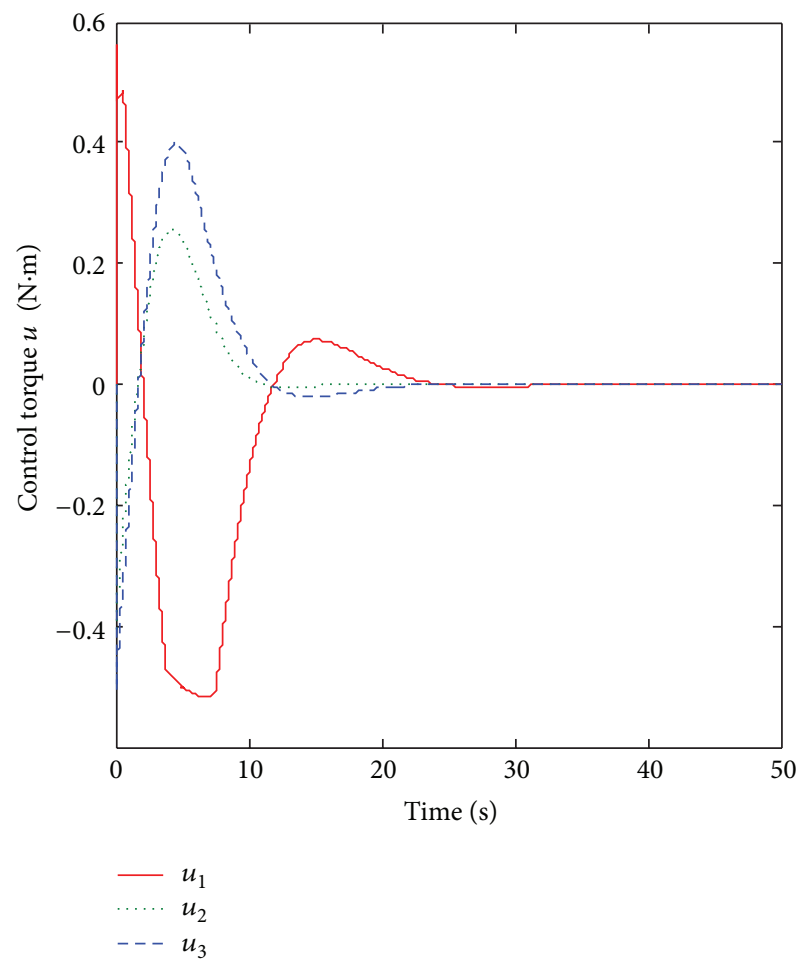

FIGURE 5: Time response of control torque.

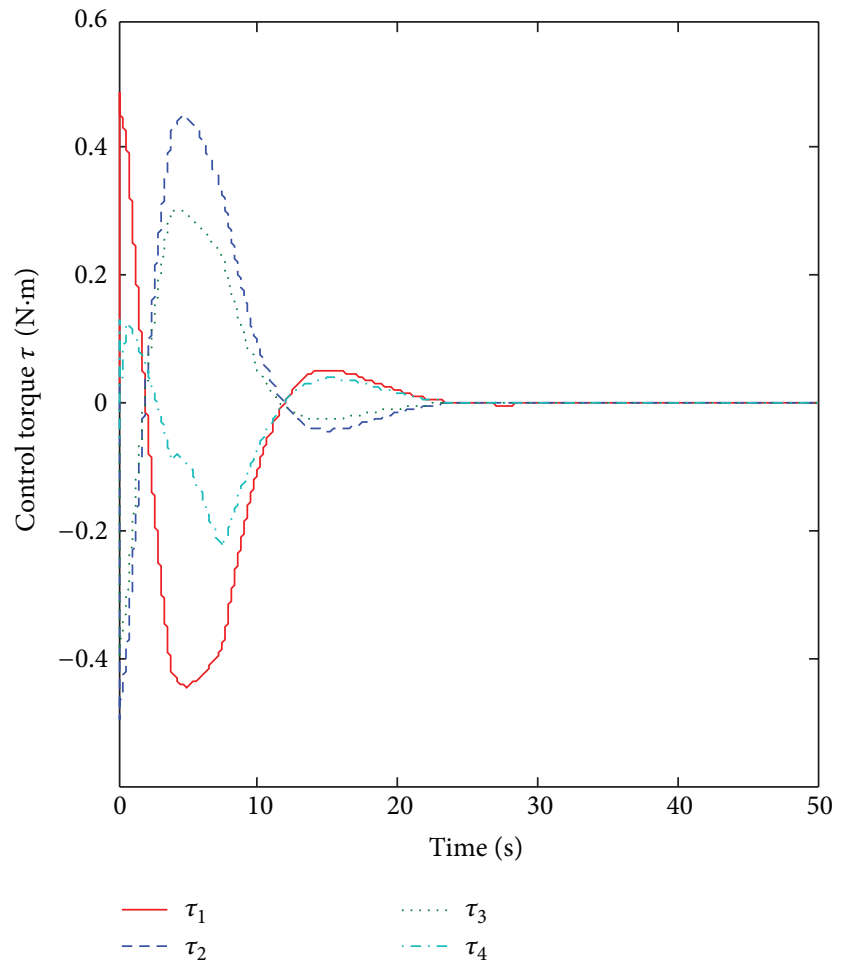

FIGURE 6: Time response of control torque for reaction wheels. 


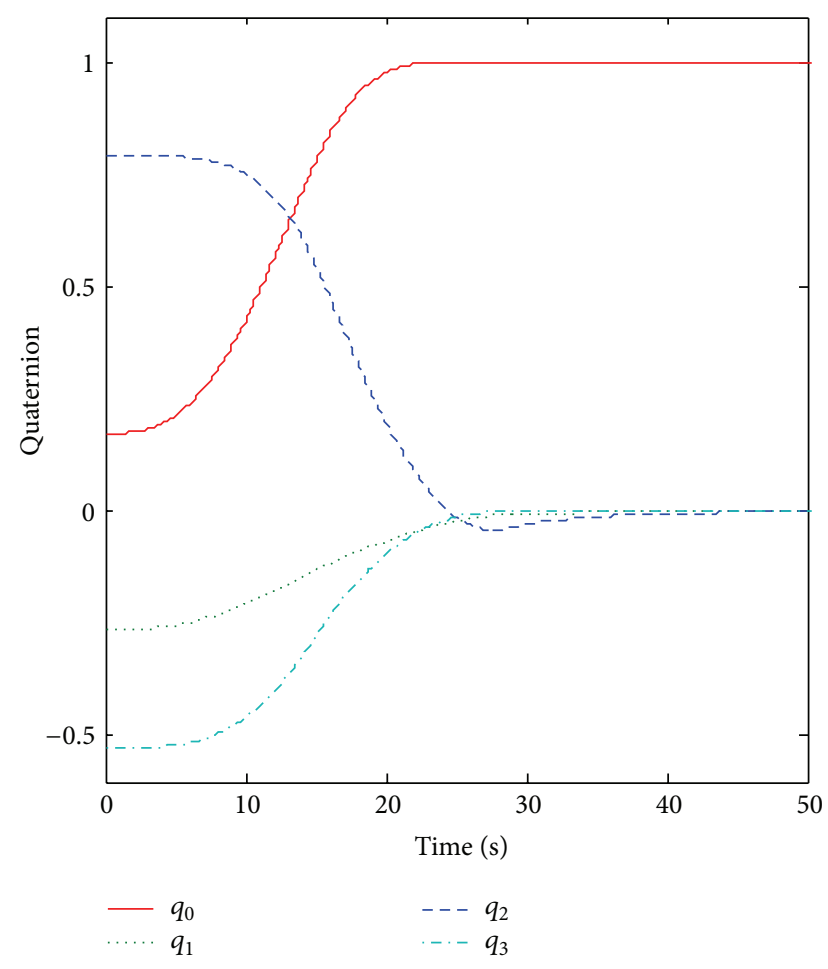

Figure 7: Time responses of quaternion.

we can see that for compensating the misalignment and other uncertainties, the designed control command of control redundancy configuration for 4 reaction wheels $\boldsymbol{\tau}$ is allocated to the three-actual-output torque $\mathbf{u}$, and then the purpose of compensation, attitude high precision control is realized. In addition, the finite-time control validity is shown in Figure 8. And from Figure 8, we can see that the spacecraft attitude control system status has realized the tracking control at $t_{F}=30.5$. Thereinto, the spacecraft attitude has arrived at slide mode surface $\mathbf{s}$ at $t_{F 1}=25.9$; afterwards, the statuses converge to equilibrium point at $t_{F 2}=5.4$ under the normal control $\boldsymbol{\tau}_{\text {nom }}(t)$. The same validity of finite-time attitude compensation control strategy proposed in this paper can be further proved from the time response of the quaternion as shown in Figure 7.

From the above illustrated simulation results, it is shown that the proposed scheme can accomplish the attitude stabilization in finite-time in presence of time-varying external disturbances, uncertain inertial parameters, and even reaction wheel installation deviation.

\section{Conclusions and Future Works}

Considering the spacecraft issues about reaction misalignment, external disturbances, and parameters uncertainty, in this paper, a finite-time adaptive attitude compensation control has been proposed. A quantitative installation deviation angle analysis has been done and given out the value range of the reaction wheel misalignment angle. In the end the system stability and engineering practical value have been discussed from the perspective of theory and engineering.

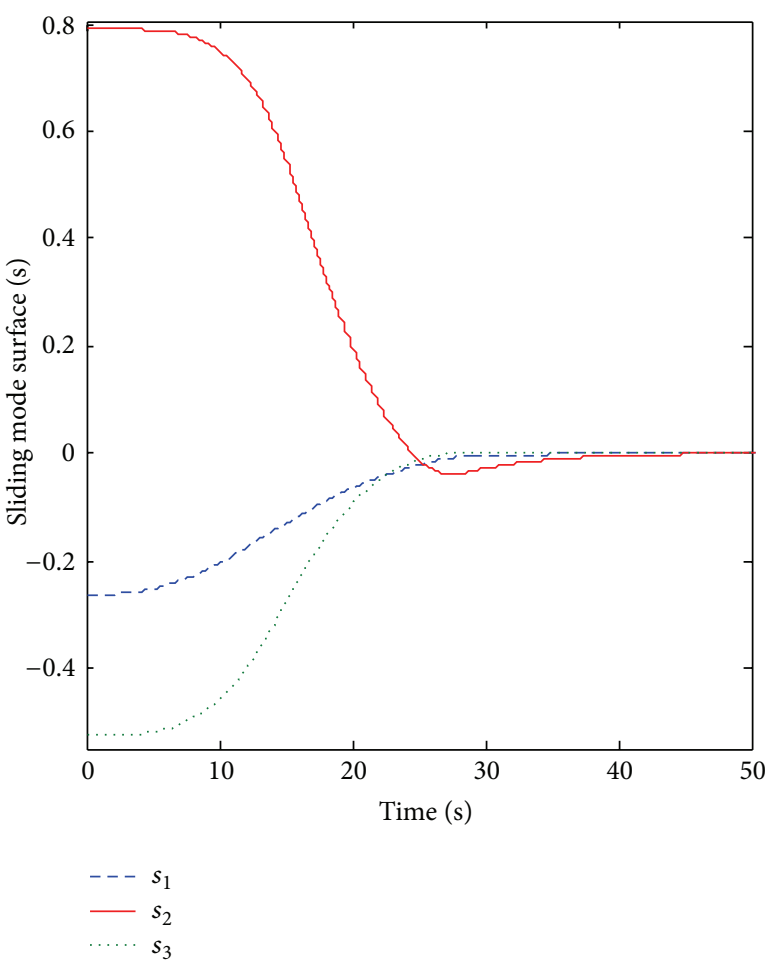

FIGURE 8: Time response of sliding mode surface.

Numerical simulation of this novel control strategy was also presented to confirm the advantages and improvements over existing controllers. The case of actuator misalignment mentioned in Section 4 had only discussed for four reaction wheel configuration, but this compensation control scheme is suitable for more than that reaction wheel number. Moreover, the actuator faults have not been considered. The latter case should be as one of subjects for future research. Meanwhile, the method optimal control approach combined robust control $[37,38]$ also can be applied in this field.

\section{Conflict of Interests}

The authors declare that there is no conflict of interests regarding the publication of this paper.

\section{Acknowledgments}

The present work was supported partially by the National Natural Science Foundation of China (Project no. 61304149) and Natural Science Foundation of Liaoning, China (Project no. 2013020044). The authors highly appreciate the above financial supports.

\section{References}

[1] B. Xiao, Q. Hu, and Y. Zhang, "Fault-tolerant attitude control for flexible spacecraft without angular velocity magnitude measurement," Journal of Guidance, Control, and Dynamics, vol. 34, no. 5, pp. 1556-1561, 2011. 
[2] H. Wong, M. S. de Queiroz, and V. Kapila, "Adaptive tracking control using synthesized velocity from attitude measurements," Automatica, vol. 37, no. 6, pp. 947-953, 2001.

[3] J. L. Junkins, M. R. Akella, and R. D. Robinett, "Nonlinear adaptive control of spacecraft maneuvers," Journal of Guidance, Control, and Dynamics, vol. 20, no. 6, pp. 1104-1110, 1997.

[4] S. R. Vadali, "Variable structure control of spacecraft large attitude maneuvers," Journal of Guidance, Control, and Dynamics, vol. 9, no. 2, pp. 235-239, 1986.

[5] S.-C. Lo and Y.-P. Chen, "Smooth sliding-mode control for spacecraft attitude tracking maneuvers," Journal of Guidance, Control, and Dynamics, vol. 18, no. 6, pp. 1345-1349, 1995.

[6] L. G. Wu, W. X. Zheng, and H. J. Gao, "Dissipativity-based sliding mode control of switched stochastic systems," IEEE Transactions on Automatic Control, vol. 58, no. 3, pp. 785-793, 2013.

[7] L. Wu, X. Su, and P. Shi, "Sliding mode control with bounded L2 gain performance of Markovian jump singular time-delay systems," Automatica, vol. 48, no. 8, pp. 1929-1933, 2012.

[8] C.-D. Yang and Y.-P. Sun, "Mixed $\mathrm{H}_{2} / \mathrm{H}_{\infty}$ state-feedback design for microsatellite attitude control," Control Engineering Practice, vol. 10, no. 9, pp. 951-970, 2002.

[9] Q. Zheng and F. Wu, "Nonlinear $\mathrm{H}_{\infty}$ control designs with axisymmetric spacecraft control," Journal of Guidance, Control, and Dynamics, vol. 32, no. 3, pp. 850-859, 2009.

[10] C. K. Carrington and J. L. Junkins, "Optimal nonlinear feedback control for spacecraft attitude maneuvers," Journal of Guidance, Control, and Dynamics, vol. 9, no. 1, pp. 99-107, 1986.

[11] W. Luo, Y.-C. Chu, and K.-V. Ling, "Inverse optimal adaptive control for attitude tracking of spacecraft," IEEE Transactions on Automatic Control, vol. 50, no. 11, pp. 1639-1654, 2005.

[12] X. Su, P. Shi, L. Wu, and Y.-D. Song, "A novel control design on discrete-time Takagi-Sugeno fuzzy systems with time-varying delays," IEEE Transactions on Fuzzy Systems, vol. 21, no. 4, pp. 655-671, 2013.

[13] S. Yin, H. Luo, and S. Ding, "Real-time implementation of faulttolerant control systems with performance optimization," IEEE Transactions on Industrial Electronics, vol. 61, no. 5, pp. 24022411, 2013.

[14] S. Yin, A. A. Sari, S. X. Ding, and H. Hao, "Data-driven monitoring for stochastic systems and its application on batch process," International Journal of Systems Science, vol. 44, no. 7, pp. 1366-1376, 2013.

[15] S. Yin, S. X. Ding, H. Hao, A. Haghani, and P. Zhang, "A comparison study of basic data-driven fault diagnosis and process monitoring methods on the benchmark Tennessee Eastman process," Journal of Process Control, vol. 22, no. 9, pp. 1567-1581, 2012.

[16] S. Yin, X. Yang, and H. R. Karimi, "Data-driven adaptive observer for fault diagnosis," Mathematical Problems in Engineering, vol. 2012, Article ID 832836, 21 pages, 2012.

[17] H. Yoon and P. Tsiotras, "Adaptive spacecraft attitude tracking control with actuator uncertainties," in Proceedings of the AIAA Guidance, Navigation, and Control Conference, pp. 5311-5322, San Francisco, Calif, USA, August 2005.

[18] P. P. Biswas, S. Ray, and A. N. Samanta, "Nonlinear control of high purity distillation column under input saturation and parametric uncertainty," Journal of Process Control, vol. 19, no. 1, pp. 75-84, 2009.

[19] M. A. Peck, "Estimation of wheel and CMG alignments from on-orbit telemetry," in Proceedings of the Flight Mechanics Symposium, 2001.
[20] H.-C. Lim and H. Bang, "Adaptive control for satellite formation flying under thrust misalignment," Acta Astronautica, vol. 65, no. 1-2, pp. 112-122, 2009.

[21] S. Ding and S. Li, "Stabilization of the attitude of a rigid spacecraft with external disturbances using finite-time control techniques," Aerospace Science and Technology, vol. 13, no. 4-5, pp. 256-265, 2009.

[22] E. Jin and Z. Sun, "Robust controllers design with finite time convergence for rigid spacecraft attitude tracking control," Aerospace Science and Technology, vol. 12, no. 4, pp. 324-330, 2008.

[23] M. Zhihong, A. P. Paplinski, and H. R. Wu, "Robust MIMO terminal sliding mode control scheme for rigid robotic manipulators," IEEE Transactions on Automatic Control, vol. 39, no. 12, pp. 2464-2469, 1994.

[24] Y. Feng, X. Yu, and Z. Man, "Non-singular terminal sliding mode control of rigid manipulators," Automatica, vol. 38, no. 12, pp. 2159-2167, 2002.

[25] A.-M. Zou, K. D. Kumar, Z.-G. Hou, and X. Liu, "Finite-time attitude tracking control for spacecraft using terminal sliding mode and chebyshev neural network," IEEE Transactions on Systems, Man, and Cybernetics B, vol. 41, no. 4, pp. 950-963, 2011.

[26] S. Wu, G. Radice, Y. Gao, and Z. Sun, "Quaternion-based finite time control for spacecraft attitude tracking," Acta Astronautica, vol. 69, no. 1-2, pp. 48-58, 2011.

[27] B. Xiao, Q. Hu, and Y. Zhang, "Adaptive sliding mode fault tolerant attitude tracking control for flexible spacecraft under actuator saturation," IEEE Transactions on Control Systems Technology, vol. 20, no. 6, pp. 1605-1612, 2012.

[28] Q. Hu, B. Xiao, and M. I. Friswell, "Robust fault-tolerant control for spacecraft attitude stabilisation subject to input saturation," IET Control Theory and Applications, vol. 5, no. 2, pp. 271-282, 2011.

[29] Z. Meng, W. Ren, and Z. You, "Distributed finite-time attitude containment control for multiple rigid bodies," Automatica, vol. 46, no. 12, pp. 2092-2099, 2010.

[30] M. J. Sidi, Spacecraft Dynamics and Control, Cambridge University Press, Cambridge, UK, 1997.

[31] S. Abramsky and L. Hardy, "Logical bell inequalities," Physical Review A, vol. 85, no. 6, Article ID 062114, 2012.

[32] S. Yu, X. Yu, B. Shirinzadeh, and Z. Man, "Continuous finitetime control for robotic manipulators with terminal sliding mode," Automatica, vol. 41, no. 11, pp. 1957-1964, 2005.

[33] T. I. Fossen and T. A. Johansen, "A survey of control allocation methods for ships and underwater vehicles," in Proceedings of the 14th Mediterranean Conference on Control and Automation (MED '06), June 2006.

[34] J. T.-Y. Wen and K. Kreutz-Delgado, "The attitude control problem," IEEE Transactions on Automatic Control, vol. 36, no. 10, pp. 1148-1162, 1991.

[35] W. Cai, X. H. Liao, and Y. D. Song, "Indirect robust adaptive fault-tolerant control for attitude tracking of spacecraft," Journal of Guidance, Control, and Dynamics, vol. 31, no. 5, pp. 1456-1463, 2008.

[36] Y. D. Song and W. Cai, "Quaternion observer-based modelindependent attitude tracking control of spacecraft," Journal of Guidance, Control, and Dynamics, vol. 32, no. 5, pp. 1476-1482, 2009. 
[37] X. Gao, K. L. Teo, and G. Duan, "An optimal control approach to robust control of nonlinear spacecraft rendezvous system with q-D technique," International Journal of Innovative Computing, Information and Control, vol. 9, no. 5, pp. 2099-2110, 2013.

[38] S. Yoon, S. J. Lee, B. Lee, C. J. Kim, Y. J. Lee, and S. Sung, “Design and flight test of a small tri-rotor unmanned vehicle with a LQR based onboard attitude control system," International Journal of Innovative Computing, Information and Control, vol. 9, no. 6, pp. 2347-2360, 2013. 


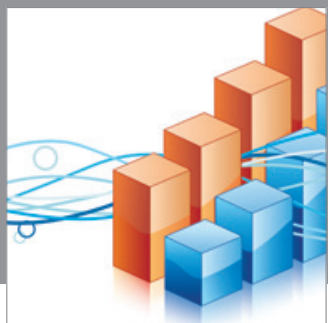

Advances in

Operations Research

mansans

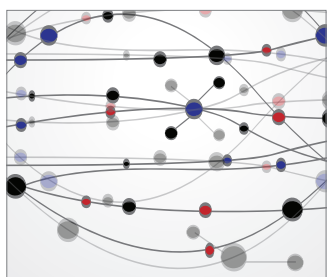

The Scientific World Journal
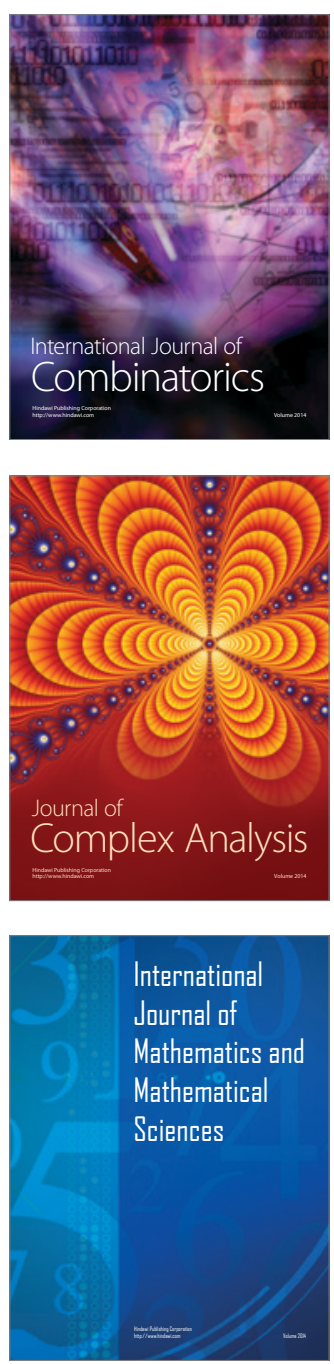
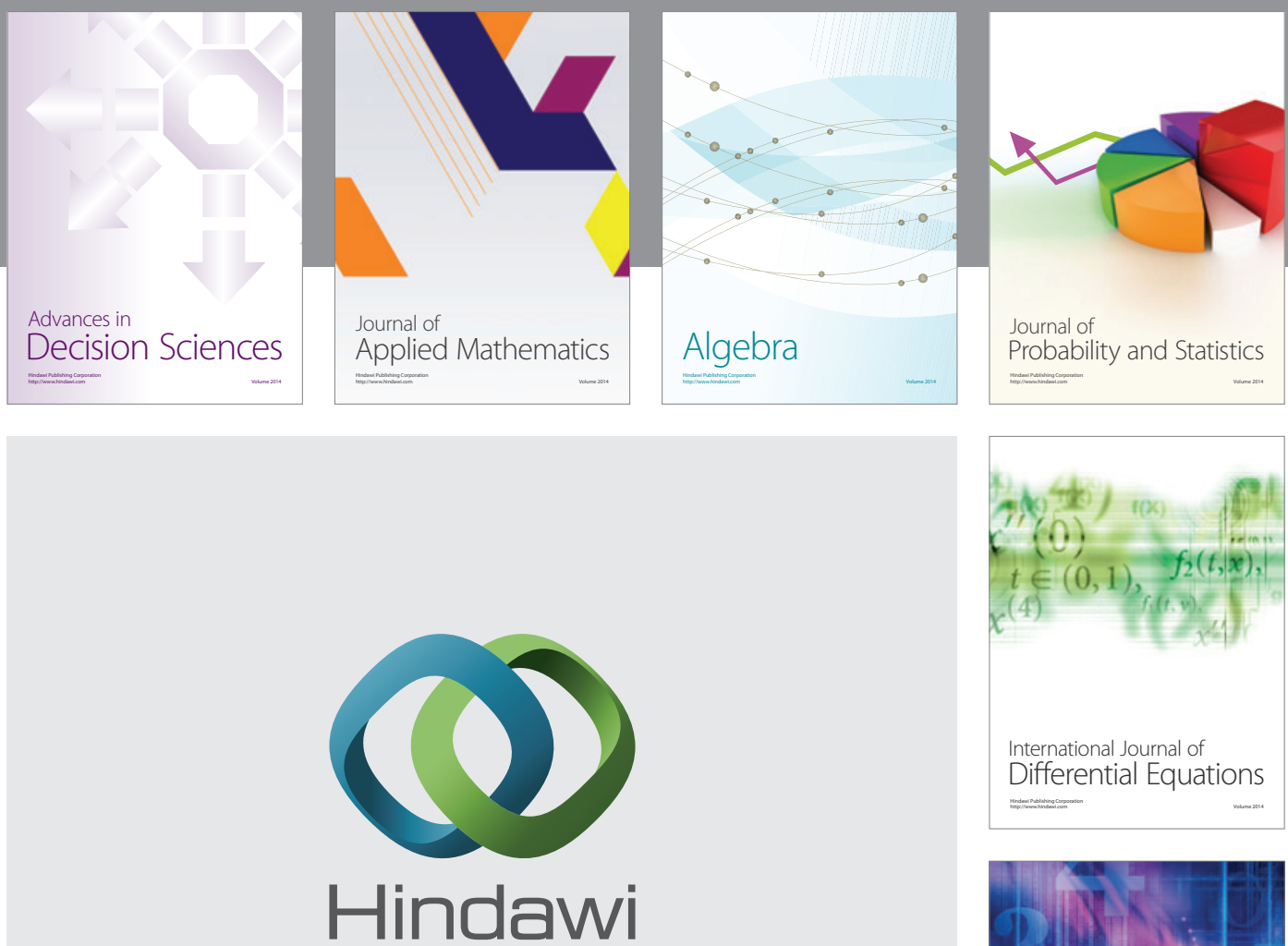

Submit your manuscripts at http://www.hindawi.com
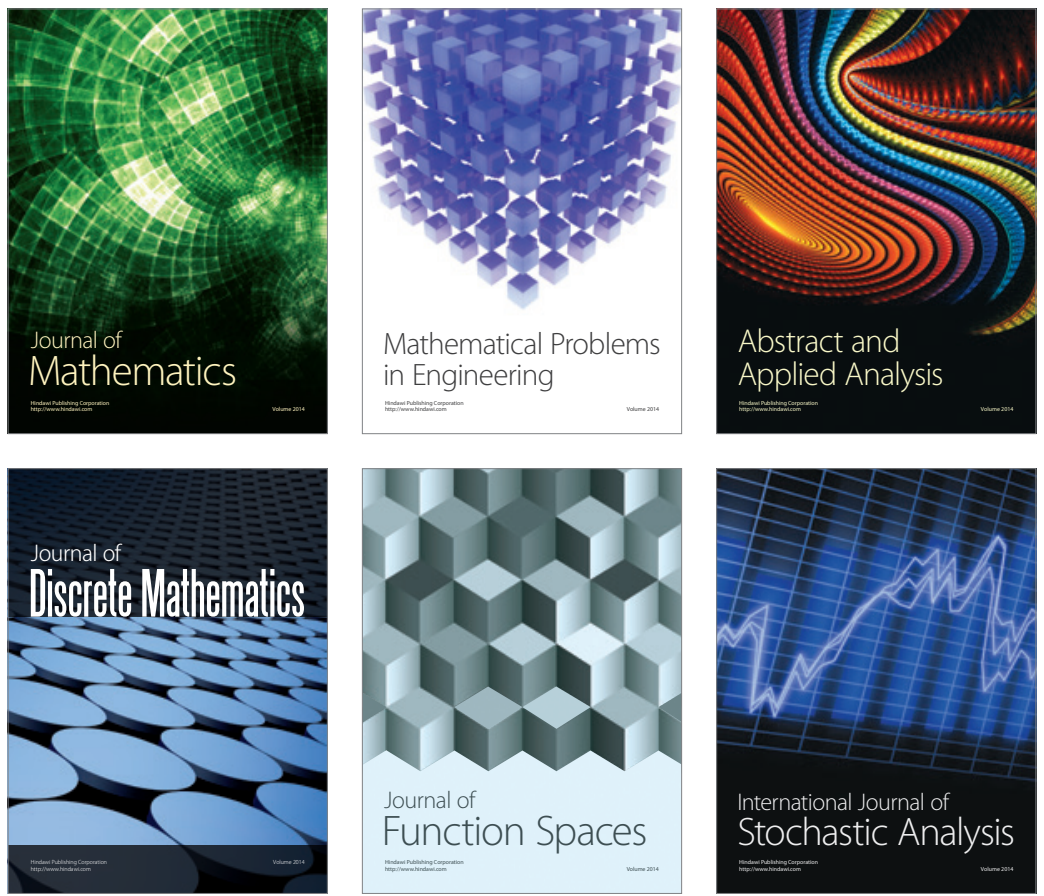

Journal of

Function Spaces

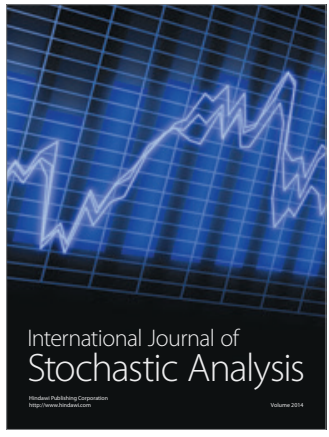

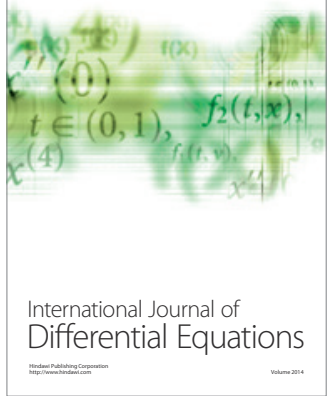
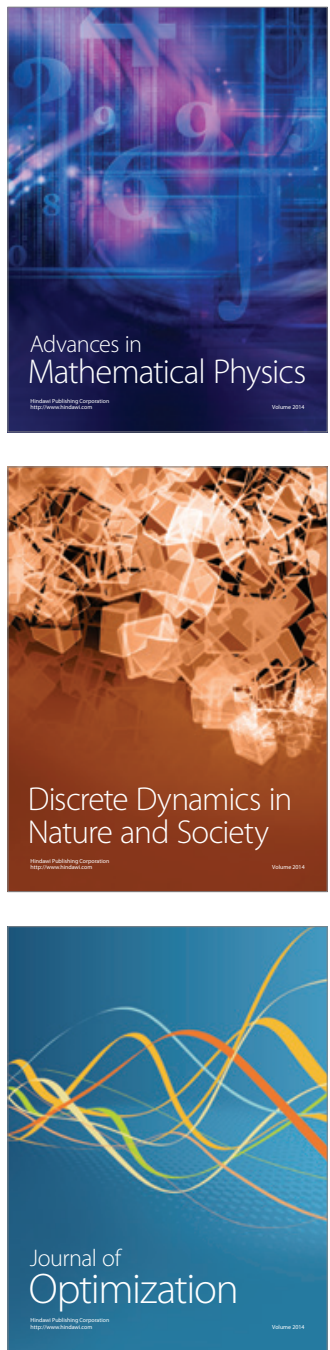